\title{
AN ADAPTIVE KALMAN FILTER FOR THE ENHANCEMENT OF NOISY AR SIGNALS
}

\author{
Gerhard Doblinger \\ Institut für Nachrichtentechnik und Hochfrequenztechnik \\ Vienna University of Technology, Gußhausstraße 25/E389, A-1040 Vienna Austria \\ gerhard.doblinger@tuwien.ac.at
}

\begin{abstract}
In this paper, we describe a new adaptive system for the enhancement of autoregressive (AR) signals which are disturbed by additive broadband noise. The system is comprised of an adaptive Kalman filter operating as a fixed-lag smoother and a subsystem for AR parameter estimation. As opposed to the conventional approach of employing an extended Kalman filter, we estimate the Kalman filter parameters using the enhanced signal and thus establishing a feedback between the Kalman filter output and the estimated parameters. Our system is capable of tracking short-time stationary signals. It is computationally efficient and can easily be implemented on today's integrated digital signal processors.
\end{abstract}

\section{INTRODUCTION}

Many natural signals can be sufficiently represented by autoregressive (AR) models. Examples are audio signals like speech and music, seismic signals, and biomedical signals. In many applications, however, these signals are corrupted by additive broadband noise (e.g. measurement noise, environmental noise) and thus signal enhancement must be applied prior to further processing of these signals.

Because of its high flexibility, the Kalman filter is widely used for signal enhancement. The key point for the performance of such a signal enhancement system is the estimation of the AR parameters in the presence of noise. Since the AR model is built into the structure of the Kalman filter, we must ensure that the estimated AR parameters yield stable filters. For this reason, we have investigated two recently published and computationally efficient methods to obtain AR parameters of noisy signals $[1,2]$. The first technique makes use of the fact that the covariance matrix of the enhanced signal may be obtained - in principle - by subtracting the diagonal noise covariance matrix from the noisy AR signal covariance matrix [1]. Unfortunately, this compensation method is very sensitive with respect to covariance matrix estimation errors, and according to our experience delivers unstable AR filters in most cases. Thus, the Kalman filter tends to be unstable, even at moderate noise levels (signal-to-noise ratio SNR $=10 \ldots 15 \mathrm{~dB}$ ). The second approach involves an adaptive transversal filter with a special LMS algorithm for improved AR parameter estimation of noisy signals [2]. Again, stability is not guaranteed by this algorithm. In addition, convergence time is very large which in turn results in a large settling period of the Kalman filter.

The most promising method is given by the extended Kalman filter which works very well at moderate noise levels [3]. However, for SNRs less than $10 \mathrm{~dB}$ this algorithm exhibits convergence problems, i.e. a stalling effect may be observed. The stalling phenomenon results in intermittent signal segments and in slow convergence (as compared to the convergence speed at higher SNRs).

In this paper, we describe a different enhancement algorithm based on an adaptive Kalman filter (fixed-lag smoother) which avoids the aforementioned problems and which also requires a minimum of a priori parameters. The novelty of our approach is the proper combination of a Kalman filter and a parameter estimation procedure which allows for tracking of nonstationary signals. We first present a computationally efficient set of Kalman fixed-lag equations tailored for use with AR signal models. Afterwards, we show the block diagram of our adaptive system and discuss its operation principle, convergence and performance behavior in some detail. Finally, we present typical experimental results and an illustrative example.

\section{KALMAN FIXED-LAG SMOOTHER FOR AR SIGNAL MODELS}

Throughout this paper, we represent the desired signal $s(n)$ by a $p^{t h}$ order AR process:

$$
\begin{aligned}
s(n+1) & =\sum_{k=1}^{p} a_{k}(n) s(n-k+1)+u(n) \\
& =\mathbf{a}^{T}(n) \mathbf{s}(n)+u(n),
\end{aligned}
$$


where $\mathbf{a}(n)=\left(a_{1}(n), \ldots, a_{p}(n)\right)^{T}$ denotes the time dependent AR parameter vector and $\mathrm{s}(n)=(s(n), \ldots, s(n-$ $p+1))^{T}$ is the signal vector. The driving noise sequence $u(n)$ of the AR model is assumed to be zero mean white Gaussian noise with variance $\sigma_{u}^{2}(n)$. Observations $y(n)$ of the desired signal $s(n)$ may be contaminated according to

$$
y(n)=s(n)+v(n),
$$

where the disturbing noise $v(n)$ is uncorrelated to $s(n)$ and is zero mean white Gaussian noise with variance $\sigma_{v}^{2}(n)$.

In practice, a fixed model order $p$ in the range of $8 \ldots 20$ may be selected. We assume that only the variance $\sigma_{v}^{2}(n)$ of the disturbing noise must be known in advance. All other parameters are supplied by our adaptive algorithm. Additionally, it turns out that the algorithm is relatively robust with regard to estimation errors of $\sigma_{v}^{2}(n)$. Thus, a precise measurement of the disturbing noise variance is not necessary.

If the parameter set $\left\{\mathbf{a}(n), \sigma_{u}^{2}(n), \sigma_{v}^{2}(n)\right\}$ of the signal model is known, then we can design a Kalman filter which optimally suppresses the disturbing noise $v(n)$. We prefer to use a Kalman fixed-lag smoother instead of the Kalman filter, since smoothing involves additional data in the estimation procedure. As a consequence, the estimation error variance is further decreased, and with a large lag length (delay) the performance approaches that of a noncausal Wiener filter for stationary signals [4].

In order to obtain a Kalman fixed-lag smoother we represent the AR signal model (1) in state-space form

$$
\mathbf{s}(n+1)=\mathbf{A}(n) \mathbf{s}(n)+(u(n), 0, \ldots, 0)^{T} .
$$

In contrast to $(1)$, the $d+1$ dimensional state vector $\mathbf{s}(n)$ now consists of the current signal sample $s(n)$ and $d$ delayed samples where $d \geq p$ is the smoother delay. Comparison of the state-space model (3) with the scalar signal model (1) immediately unveils the following structure of the $(d+1) \times(d+1)$ state matrix $\mathbf{A}(n)$ :

$$
\mathbf{A}(n)=\left(\begin{array}{ccccccc}
a_{1}(n) & \cdots & a_{p}(n) & 0 & \cdots & 0 & 0 \\
1 & \cdots & 0 & 0 & \cdots & 0 & 0 \\
\vdots & \ddots & & & & & \vdots \\
0 & & 1 & 0 & \cdots & 0 & 0 \\
0 & & 0 & 1 & \cdots & 0 & 0 \\
\vdots & & & & \ddots & & \vdots \\
0 & \cdots & 0 & 0 & \cdots & 1 & 0
\end{array}\right) .
$$

Having established the state-space signal model we may formulate the optimal state estimation as a Kalman filter:

$$
\begin{aligned}
& \mathbf{x}(n+1)=\mathbf{A}(n) \mathbf{x}(n)+\mathbf{k}(n)\left(y(n)-\mathbf{a}^{T}(n) \mathbf{x}_{p}(n)\right) \\
& \hat{s}(n-d)=x_{d+1}(n),
\end{aligned}
$$

where $\mathbf{x}(n)=\left(x_{1}(n), \ldots, x_{p}(n), \ldots, x_{d+1}(n)\right)^{T}$ is the Kalman filter state vector which in turn is the estimate of the desired signal vector $\mathbf{s}(n)$. Vector $\mathbf{x}_{p}(n)$ contains the first $p$ components of $\mathbf{x}(n)$. The enhanced signal sample $\hat{s}(n-d)$ is given by the state vector component with index $d+1$.

The update equations for the Kalman fixed-lag smoother can be simplified due to the special structure of state matrix $\mathbf{A}(n)$. In the following equations, we use a notation for submatrices which can be easily converted to MATLAB syntax:

If $\mathbf{P}=\mathbf{P}_{(1: d, 1: d)}$ is a $d \times d$ matrix, then we will use

$\mathbf{P}_{(1: p, 1: q)}$ to denote the $p \times q$ submatrix containing the first $p$ rows and first $q$ columns of $\mathbf{P}$,

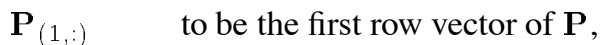

$\mathbf{P}_{(:, 1)} \quad$ to be the first column vector of $\mathbf{P}$,

$\mathbf{P}_{(1,1)} \quad$ to be the first element of $\mathbf{P}$, etc.

A similar notational convention will be used for subvectors and single vector components. Therefore, (5) reads as

$$
\begin{aligned}
\tilde{\mathbf{x}}(n) & =\left(\begin{array}{c}
\mathbf{a}^{T}(n) \mathbf{x}_{(1: p)}(n) \\
\mathbf{x}_{(1: d)}(n)
\end{array}\right) \\
\mathbf{x}(n+1) & =\tilde{\mathbf{x}}(n)+\mathbf{k}(n)\left(y(n)-\tilde{\mathbf{x}}_{(1)}(n)\right) \\
\hat{s}(n-d) & =\mathbf{x}_{(d+1)}(n)
\end{aligned}
$$

Modifying the general Kalman equations as given e.g. in [5] we obtain:

$$
\begin{aligned}
\mathbf{p}^{T}(n) & =\mathbf{a}^{T}(n) \mathbf{P}_{(1: p, 1: d)}(n) \\
\widetilde{\mathbf{P}}(n) & =\left(\begin{array}{cc}
\mathbf{p}_{(1: p)}^{T}(n) \mathbf{a}(n)+\hat{\sigma}_{u}^{2}(n) & \mathbf{p}^{T}(n) \\
\mathbf{p}(n) & \mathbf{P}_{(1: d, 1: d)}(n)
\end{array}\right) \\
\mathbf{k}(n) & =\frac{\widetilde{\mathbf{P}}_{(:, 1)}(n)}{\widetilde{\mathbf{P}}_{(1,1)}(n)+\hat{\sigma}_{v}^{2}(n)} \\
\mathbf{P}(n+1) & =\widetilde{\mathbf{P}}(n)-\mathbf{k}(n) \widetilde{\mathbf{P}}_{(1,:)}(n)
\end{aligned}
$$

The Kalman filter variables may be initialized by

$$
\begin{aligned}
\mathbf{P}_{(1,1)}(0) & =\hat{\sigma}_{u}^{2}(0) \\
\mathbf{P}_{(2: d+1,2: d+1)}(0) & =\mathbf{0} \\
\mathbf{x}_{(1: d+1)}(0) & =\mathbf{0} .
\end{aligned}
$$

Both the state matrix $\mathbf{A}(n)$ and the Kalman gain vector $\mathbf{k}(n)$ depend on the signal model parameters and thus the Kalman filter will track nonstationary signals.

\section{OPERATION PRINCIPLE OF THE ADAPTIVE KALMAN FILTER}

The block diagram of our signal enhancement system is shown in Fig. 1. The enhanced signal at the output of the Kalman filter is fed to an AR parameter estimation subsystem. At the beginning of the system's convergence period 
the noisy signal is used for AR parameter estimation. However, after convergence the parameter estimation is carried out on the de-noised signal. Convergence of the system is ensured as long as the AR signal contains signal components which are stronger than the disturbing noise. Since the Kalman filter operates as an optimum filter, it emphasizes strong signal components. Due to the feedback loop the parameter estimation of the AR signal is further improved.

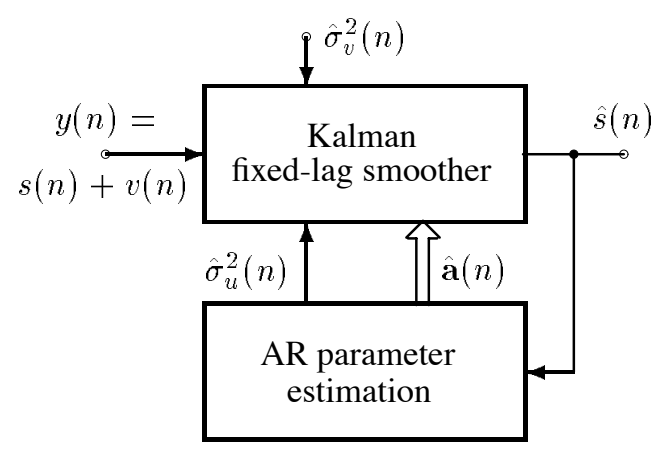

Figure 1: Block diagram of the adaptive Kalman filter for the enhancement of AR signal $s(n)$ disturbed by noise $v(n)$.

We have selected and compared two algorithms for the AR parameter estimation subsystem in Fig. 1. Method I is a block processing technique where the block length and overlapping is chosen in accordance to the nonstationarity of the AR signal. It uses correlation function estimation and the Levinson algorithm to obtain $\hat{\mathbf{a}}$ and $\hat{\sigma}_{u}^{2}$. The details can be found e.g. in [6]. Method II uses a recursive least-squares lattice (LSL) algorithm and operates on a sample per sample basis [7]. We use an exponential error weighting for a gentle fit of the LSL algorithm to nonstationary signals.

Both methods show a comparable computational complexity and performance behavior. However, application of method I (based on the Levinson algorithm) guarantees stability of the AR model. In general, the recursive LSL algorithm with exponential error weighting used in method II may produce unstable AR filters. However, stability can be easily checked by observing the reflection coefficients of the LSL algorithm. In addition, in all our experiments with a wide range of AR signals we have never encountered any stability problems.

The estimation of $\sigma_{u}^{2}$ is a crucial point since its value determines the noise reduction behavior and the convergence speed of the adaptive Kalman filter. Furthermore, we must use a lower bound $\sigma_{\min }^{2} \neq 0$ for $\hat{\sigma}_{u}^{2}$ since $\hat{\sigma}_{u}^{2}=0$ prevents a proper start up of the algorithm (see (11) and (14)). If $\sigma_{\text {min }}^{2}$ is chosen too small, then the signal enhancement is certainly better but convergence is slow in general, and vice versa. It should be noted that the estimate $\hat{\sigma}_{u}^{2}$ is less reliable when using method II because of the exponential error weighting of the LSL algorithm.

\section{PERFORMANCE ANALYSIS}

The performance of the system shown in Fig. 1 lies in between two limiting cases. The ideal case will occur, if the AR parameters are known exactly and thus we can achieve the performance of the standard (non-adaptive) Kalman smoother. The worst case will arise, if we use the noisy input data to estimate the AR parameters.

In the sequel, we assume stationary signals disturbed by additive zero mean white Gaussian noise to present a simplified error analysis of our system. Our goal is to derive an upper bound of the AR parameter error vector since that error limits the performance of our system. Therefore, we first investigate how additive noise affects an AR parameter estimation in principle.

If the noisy signal is given by $y(n)=s(n)+w(n)$, then its $p \times p$ correlation matrix $\mathbf{R}_{y}$ is

$$
\mathbf{R}_{y}=\mathbf{R}_{s}+\sigma_{w}^{2} \mathbf{I}
$$

where $\sigma_{w}^{2}$ is the noise variance and $\mathbf{I}$ is the identity matrix. The true AR vector a can be found as the solution of $\mathbf{R}_{s} \mathbf{a}=\mathbf{r}_{s}$ whereas a (estimated with the noisy data) is obtained be solving $\mathbf{R}_{y} \hat{\mathbf{a}}=\mathbf{r}_{y}$. For white noise disturbances the correlation vectors $\mathbf{r}_{s}$ and $\mathbf{r}_{y}$ are equal since they both contain the correlations $r_{s}(1), r_{s}(2), \ldots, r_{s}(p)$. Combination of these facts with (17) yields

$$
\hat{\mathbf{a}}=\left(\mathbf{I}-\sigma_{w}^{2} \mathbf{R}_{y}^{-1}\right) \mathbf{a} .
$$

Using a vector (matrix) norm, the relative error regarding the AR coefficient vector can be expressed by

$$
\varepsilon=\frac{\|\mathbf{a}-\mathbf{a}\|_{2}}{\|\mathbf{a}\|_{2}}=\sigma_{w}^{2} \frac{\left\|\mathbf{R}_{y}^{-1} \mathbf{a}\right\|_{2}}{\|\mathbf{a}\|_{2}} \leq \sigma_{w}^{2}\left\|\mathbf{R}_{y}^{-1}\right\|_{2} .
$$

The matrix norm in (19) is given by $\left\|\mathbf{R}_{y}^{-1}\right\|_{2}=1 / \lambda_{y_{\min }}$ with the eigenvalue $\lambda_{y_{\text {min }}}=\lambda_{s_{\text {min }}}+\sigma_{w}^{2}$. Thus, we finally get

$$
\varepsilon \leq \frac{\sigma_{w}^{2}}{\sigma_{w}^{2}+\lambda_{s_{\min }}}
$$

In the system shown in Fig. 1, we use the enhanced signal $\hat{s}(n)$ for AR parameter estimation and $\sigma_{w}^{2}$ in (20) is the variance of the remaining noise at the system output. Because this variance is decreased during the adaptation phase the error $\varepsilon$ in (20) will move towards a minimum. This in turn further improves the noise reduction performance of the system. After the settling period, the remaining noise variance causes the estimated AR vector to differ slightly from the true one. As a consequence, we cannot exactly achieve the performance of the idealized case where the AR parameters are known a priori. However, our experiments show that the difference in SNR gain is typically less than $0.5 \mathrm{~dB}$. 


\section{EXPERIMENTAL RESULTS}

We have tested the adaptive Kalman filter using a variety of AR signals with different model orders $p$, delays $d$, and noise variances $\sigma_{v}^{2}$. If the true model order $p_{0}$ of the desired signal is known, then the noise reduction behavior can be improved for an input $\mathrm{SNR}<3 \mathrm{~dB}$ by selecting $p>p_{0}$ as parameter of the adaptive Kalman filter (over-determined parameter estimation).

For stationary signals, the delay $d$ should be large in order to get a large data set for the smoothing operation. In practical implementations, its value will be limited mainly by the increase in computational complexity.

We illustrate the performance of our enhancement system by a typical experimental result as shown in Fig. 2. The clean signal is composed of a $p_{0}=4$ order AR signal (time interval $n=0 \ldots 999$ ) and a $p_{0}=3$ order AR signal (time interval $n=1000 \ldots 1970$ ). The SNR at the input of the adaptive Kalman filter is $5 \mathrm{~dB}$ and the resulting SNR improvement is $10.94 \mathrm{~dB}$. A fixed parameter $p=8$ was used by the enhancement system. By observing the enhanced signal trace Fig. 2 (c) the ability of our system to track the signal nonstationarity at $n=1000$ is clearly visible. It should be noted that the extended Kalman filter [3] did not converge at this high noise level.

The adaptive Kalman filter has also been implemented on an ADSP-21061 floating point signal processor for realtime operation with sampling frequencies up to $16 \mathrm{kHz}$. The computational complexity of the DSP implementation is $\mathcal{O}\left(5 d^{2}+(p+21) d+3 p\right)$ for the Kalman filter, $\mathcal{O}(2.5 p(p-1)+81 p)$ for the LSL algorithm, and $\mathcal{O}\left(p^{2}+(2 N+38) p+N\right)$ for the Levinson algorithm (AR model order $p$, Kalman smoother delay $d$, and block length $N)$.

\section{CONCLUSIONS}

We have described a robust and efficient adaptive Kalman smoother for enhancing AR signals corrupted by additive broadband noise. The system requires a minimum a priori knowledge of signal and noise parameters and is capable of tracking short-time stationary signals. As shown in an error analysis for stationary signals the signal enhancement performance is close to the optimal case where the AR parameters are known a priori. Future extensions of the algorithm will include colored noise and impulsive noise disturbances.

\section{REFERENCES}

[1] Wei Xing Zheng, "An efficient algorithm for parameter estimation of noisy AR processes", Proc. 1997 IEEE Int. Symp. ISCAS '97, Vol. 4, June 9-12, 1997, pp. 25092512.
[2] W-R. Wu, P-C. Chen, "Adaptive AR modeling in white gaussian noise", IEEE Trans. on Signal Processing, Vol. 45, May 1997, pp. 1184-1192.

[3] M. Niedźwiecki, K. Cisowski, "Adaptive scheme for elimination of broadband noise and impulsive disturbances from AR and ARMA signals", IEEE Trans. on Signal Processing, Vol. 44, March 1996, pp. 528-537.

[4] G. C. Goodwin, "Adaptive filtering prediction and control", Chapter 7, Prentice-Hall, Inc., 1984.

[5] S. M. Kay, "Fundamentals of Statistical Signal Processing: Estimation Theory", Chapter 13, Prentice-Hall, Inc., 1993.

[6] S. L. Marple Jr., "Digital Spectral analysis with applications", Chapter 7, Prentice-Hall, Inc., 1987.

[7] S. Haykin, "Adaptive filter theory", Chapter 9, PrenticeHall, Inc., 1987.

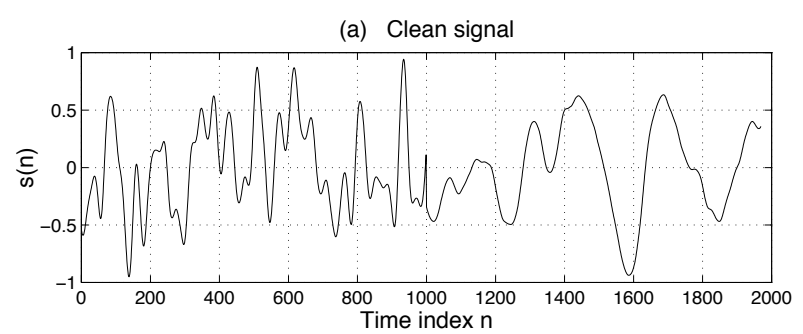

(b) Noisy signal, SNR $=5 \mathrm{~dB}$

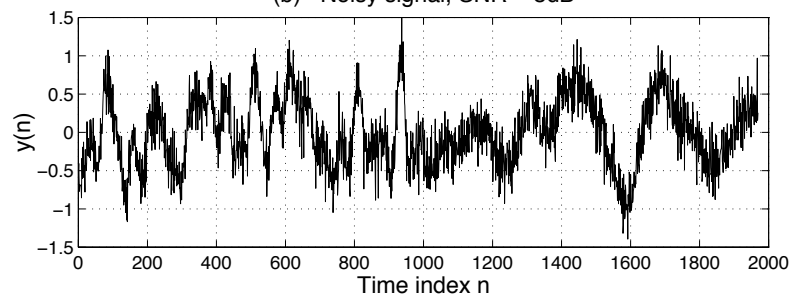

(c) Enhanced signal

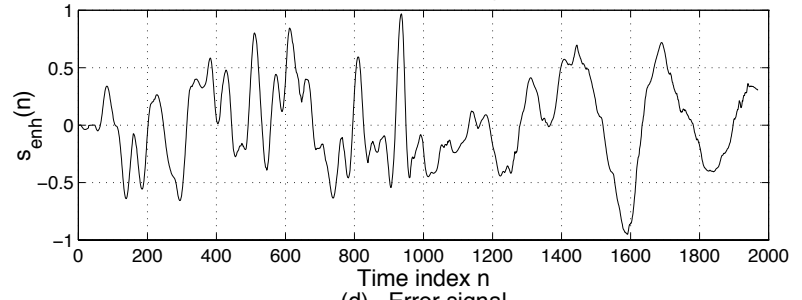

(d) Error signal

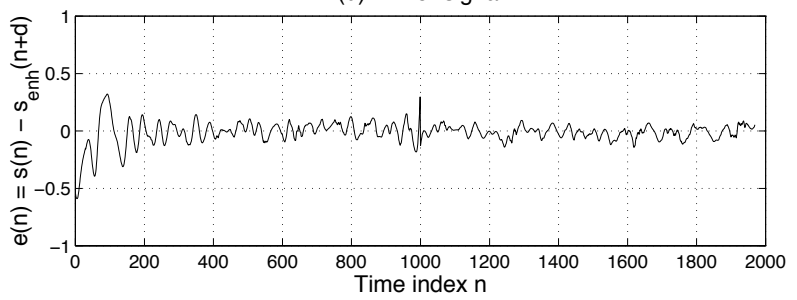

Figure 2: Signal enhancement using the adaptive Kalman filter (Levinson algorithm for AR parameter estimation with $p=8$, Kalman smoother delay $d=30$, block length $N=$ 300 , new AR parameters are computed every 10 samples, lower bound of $\hat{\sigma}_{u}^{2}$ set to $\sigma_{\min }^{2}=10^{-4}$ ). 